\title{
Parasitemia Density Of White Mice Vaccinated of Anopheles Sundaicus Salivary Gland Extract And Plasmodium Berghei Infected As A Model In Fighting Malaria
}

\author{
Adrial $^{1}$, Ellyza Nasrul ${ }^{2}$, Rosfita Rasyid ${ }^{3}$ \\ \{adrial@med.unand.ac.id ${ }^{1}$ \} \\ Department of Parasitology, Faculty of Medicine, Universitas Andalas Padang, Indonesia ${ }^{1}$, \\ Department of Clinical Pathology, Faculty of Medicine, Universitas Andalas Padang, Indonesia ${ }^{2}$, \\ Department of Public Health and Community Medicine, Faculty of Medicine, Universitas Andalas \\ Padang, Indonesia ${ }^{3}$
}

\begin{abstract}
This study aimed to determine parasitemia density of white mice vaccinated of Anopheles sundaicus (An. sundaicus) salivary gland extract and infected with Plasmodium berghei as a model in fighting malaria This study used experimental study design. This study samples $45 \mathrm{BALB} / \mathrm{c}$ mice aged 6-8 weeks consisted of three groups: control group (C): vaccinated with Phosphate Buffer Saline (PBS) mixed with adjuvant (IFA and CFA). Pellet group (P): vaccinated with pellets extract of An. sundaicus salivary glands mixed with adjuvant (IFA and CFA). Supernatan group (S): vaccinated with supernatant extracts of An. sundaicus salivary glands mixed with adjuvant (IFA and CFA).. The research was conducted at the Biomedical and Parasitology Laboratory of the Faculty of Medicine, Universitas Andalas Padang, Parasitology and Pharmacy Laboratory of Faculty of Medicine, Universitas Brawijaya Malang. Data analysis using one-way anova. A twotailed $P$-value of $<0.05$ was considered statistically significant. Data analysis was carried out in Stata version 14.2 (Stata Corporation). The results are are known parasitemia density was $8.81 \%$ for the control group, $7.02 \%$ for the pellet extract group and $3.35 \%$ for the supernatant extract group. The results of ANOVA statistical analysis obtained showed no significant differences $(\mathrm{P}>0.05)$ from the three treatment groups: the control group, pellet extract, and extract of the salivary gland supernatant. sundaicus. The results of this study concluded that the administration of supernatant extract and pellet extract of Anopheles sundaicus salivary glands was able to reduce the density of Plasmodium berghei parasitemia.
\end{abstract}

Keywords: Malaria, salivary glands, transmission-blocking vaccine, Anopheles sundaicus, parasitemia density.

\section{Introduction}

The main problem of malaria prevention until now is the increasing resistance of Plasmodium falciparum (P. falciparum) and Plasmodium vivax (P. vivax) to anti-malaria drugs. The right vaccine for malaria prevention is one that includes prevention for pre-erythrocytic cycles, erythrocytic cycles, and transmission processes. At this time found many antigen-based vaccines developed in malaria parasites in both pre-erythrocytic and erythrocytic cycles. The 
various types of vaccines mentioned above it appears that all approaches are still in the development stage and have not provided optimal results [1].

At this time a new approach is being taken to develop a vaccine against malaria which is more innovative and also plays a role in inhibiting the transmission of pathogens that cause malaria so that it overcomes the epidemic by developing Transmission-Blocking Vaccine (TBV). TBV is a vaccine that inhibits the spread of disease by targeting antigens from the body of a vector, one of which is the mosquito salivary gland [2]. The goal of TBV is to prevent the transmission of pathogens from infected vertebrate hosts to uninfected hosts [3]. TBV is widely used to generate antibodies to fight vector molecules involved in the development of pathogens. The development of TBV which is a vector-based vaccine is not only a new approach to disease control but also a preferred approach. Vector-based vaccines will not only protect against pathogens transmitted by vectors but also to other people who have not been infected. Recent research shows that mosquito salivary glands and other arthropods contain immunogenic substances which can give rise to an adaptive immune response that produces antibodies against the components of the salivary glands. For this reason, the mosquito salivary gland components of malaria can be targeted in the development of TBV to inhibit the transmission of malaria pathogens $[4,5]$.

The last type of vaccine, also called TBV, is one type of vaccine that inhibits the life cycle of parasites by inducing antibodies that will block parasites from developing in the mosquito's body shortly after sucking the blood of people who have been vaccinated [6]. One of the TBV candidates developed is parasite-based, with targets including antigen gametocytes expressed in male and female gametocytes and antigen ookinetes [7-10]. In recent years the development of TBV shows the development of the concept of vector-based TBV or mosquitos stage vaccine where one of the targets used is the vector salivary gland [9].

The arthropod vector salivary gland is successful in transmitting pathogens to the host because in the salivary glands it contains some vasodilators and immunomodulatory components which have activities as anticoagulants, vasodilation, anti-inflammatory and are immunosuppressive. If arthropod vector salivary glands containing immunomodulatory factors can increase pathogen infection, it is possible to control the transmission by vaccinating the host with molecules that are against proteins in the salivary glands. [8,11,12].

Repeated exposure of the salivary glands turned out to show a shift in immune response from Th2 towards Th1 which was host protective. Repeated exposure of the Anopheles stephensi malaria salivary gland was able to limit the development of Plasmodium yoelii growth in the body of experimental animals by influencing both systemic and local immune responses [13]. Other studies using salivary glands of the sand flies vector in Leishmaniasis have also shown inhibition of pathogenic growth in experimental animals [14]. This research hypothesis states that the salivary gland of malaria vector can prevent the spread of parasites so that it can be used as a candidate for the development of TBV against malaria.

\section{Methods}

\subsection{Study design, research sample}

This study used experimental study design. This study samples 45 BALB/ c mice aged 6-8 weeks consisted of three groups: control group (C): vaccinated with Phosphate Buffer Saline (PBS) mixed with adjuvant (IFA and CFA). Pellet group (P): vaccinated with pellets extract of 
An. sundaicus salivary glands mixed with adjuvant (IFA and CFA). Supernatant group (S): vaccinated with supernatant extracts of $A n$. sundaicus salivary glands mixed with adjuvant (IFA and CFA).

\subsection{Data collection technique}

An. sundaicus which used in this result of rearing of larvae collection from its breeding place in Sungai Pinang Village, Koto XI Subdistrict Tarusan, South Pesisir District. The larvae collection of Anopheles mosquitoes was carried out using larvae survey method by World Health Organization standards. The larvae obtained from their breeding sites are filtered in the laboratory to be hatched into adult mosquitoes. After mosquitoes hatch, using mosquito aspirators are transferred into paper cups, maintained and fed 5\% sucrose or fructose. Before the salivary glands were isolated, adult female mosquitoes were turned off by using chloroform and identified [15]. The research was conducted at the Biomedical and Parasitology Laboratory of the Faculty of Medicine, Universitas Andalas Padang, Parasitology and Pharmacy Laboratory of Faculty of Medicine, Universitas Brawijaya Malang.

\subsection{Salivary gland preparation}

Salivary gland dissection of female mosquitoes was carried out by the World Health Organization microdissection method [16]: (1) mosquitoes were placed under a stereo microscope on the right side of the slide, (2) the dissection needle on the left hand pressed gently on the thorax and the dissection needle in the right hand slowly pulls the head, (3) the salivary gland attached to the head (a form like a sausage, in the form of a refractile body) is cut and moved into a Phosphate Buffer Saline (PBS) solution. The isolated salivary glands are stored at $-20{ }^{\circ} \mathrm{C}$ until needed. A number of salivary glands An. sundaicus used in this study amounted to 1500 .

\subsection{Extract and vaccination preparations}

Vaccination in mice was carried out 3 times at 2-week intervals. Each time the vaccination was administered, the mice were injected with $100 \mathrm{~mL}$ of extract using $1 \mathrm{cc}$ disposable syringe subcutaneously on the outside of the femur just below the mice's cutaneous layer.

\subsection{Plasmodium berghei preparation}

P. berghei isolates were obtained from donor mice through the passage process. This mice donor has previously measured the level of parasitemia up to $15 \%$. Blood from mice donor is taken through the surgical process in the thorax section followed by intracardiac blood collection. Blood collection is carried out using a $1 \mathrm{cc}$ disposable syringe inserted in the heart. The blood taken is then inserted in the heparin vacutainer so that the blood does not freeze. The blood obtained was mixed with PBS solution until 104 dilutions were obtained. The solution was then put into a hemocytometer and then counted the number of erythrocytes (n) under a microscope with an objective magnification of $40 \mathrm{x}$.

Because there is dilution, the calculation of the number of erythrocytes $(\mathrm{a})=\mathrm{n} \mathrm{x} 10^{4} \times 10^{4}$ is obtained. After obtaining the number of erythrocytes, the dilution is calculated by the following formula: 


$$
\text { Total dilution }=\frac{\text { a } \times \text { degree of parasitemia }}{5 \times 10^{6}}
$$

The total of blood that will be taken by the formula is calculated:

$$
\text { The total of blood taken }=\frac{\text { The number of mice infected } \times 200 \mu l}{\text { Total dilution }}
$$

The blood obtained is then added with medium plus. The mixture is put into a $1 \mathrm{cc}$ disposable syringe and is ready to be injected.

\subsection{Inoculation of $P$. Berghei}

Inoculation of $\mathrm{P}$. berghei in each group of donor mice was carried out 2 weeks after III Immunization. Each mouse was injected with $200 \mu \mathrm{l}$ of diluted P. berghei isolate.

\subsection{Calculation of degrees of parasitemia}

The calculation of parasitemia density in BALB/c mice that had been infected with $P$. berghei was carried out 48 hours after infection, followed by counting on the $2^{\text {nd }}$ day until the $7^{\text {th }}$ day. Before the calculation, the preparation of blood smear was made from the tail of a mouse with Giemsa staining. Dry blood smears were then observed under a binocular microscope with an objective magnification of 100x using emersion oil. Parasitemia density was measured by counting the number of erythrocytes infected with ${ }^{\text {P. berghei }}$ every 1000 erythrocytes. The formula is as follows:

$$
\text { Degree of parasitemia }=\frac{\text { The number of infected erythrocytes }}{1000 \text { erythrocytes }} \times 100 \%
$$

\subsection{Data analysis}

Data analysis using one-way ANOVA. A two-tailed $P$-value of $<0.05$ was considered statistically significant. Data analysis was carried out in Stata version 14.2 (Stata Corporation).

\section{Results}

The degree of parasitemia is determined by calculating the number of erythrocytes infected every 1000 erythrocytes and expressed in percent (\%) [17]. The infected erythrocytes contain one or more ringforms, the walls appear to be more elastic and have a larger size than normal erythrocytes (Figure 1). 


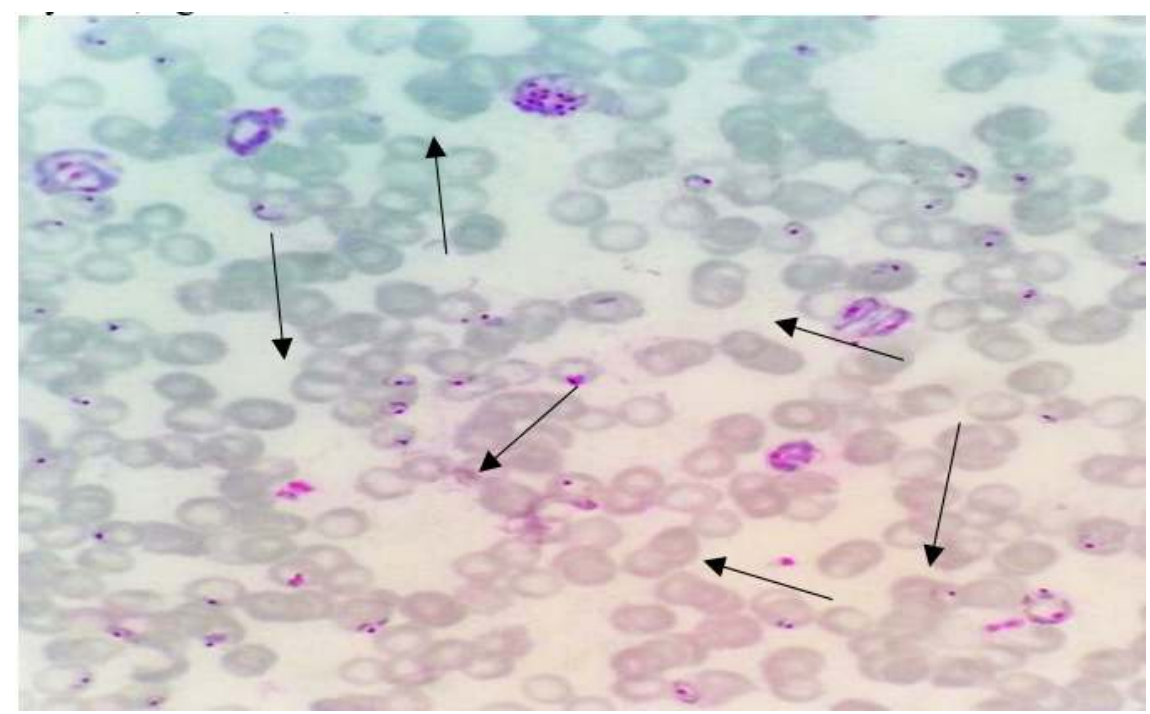

Fig. 1. Blood smear of mice after Plasmodium berghei inoculation (arrows show erythrocytes infected with parasites)

It is known that the parasitic stage morphology found during observation of the degree of mice parasitemia is trophozoite stage and schizont stage. Furthermore, it was seen from the parasitemia density. The density was increasing for the three treatment groups (Table 1).

Table 1. The density of $\mathrm{P}$. berghei, parasitemia in white mice Mus musculus Balb/ C, immunized with extracts of salivary glands An. Sundaicus

\begin{tabular}{llllllll}
\hline \multirow{2}{*}{ Groups } & \multicolumn{6}{l}{ Day / Date of Observation of Parasitemia } & \multicolumn{2}{l}{} \\
\cline { 2 - 6 } & Day 2 & Day 3 & Day 4 & Day 5 & Day 6 & Day 7 & Mean \\
\cline { 2 - 6 } & $5-31-17$ & $6-1-17$ & $6-2-17$ & $6-3-17$ & $6-4-17$ & $6-5-17$ & \\
\hline Control & 0.032 & 0.463 & 1.780 & 3.652 & 9.645 & 37.297 & $8,810^{\mathrm{a}}$ \\
Pellet Extract & 0.034 & 0.368 & 3.329 & 8.537 & 12.535 & 17.338 & $7,017^{\mathrm{a}}$ \\
Supernatan & 0 & 0.350 & 0.613 & 3.688 & 6.030 & 9.442 & $3,354^{\mathrm{a}}$ \\
Extract & & & & & & & \\
\hline
\end{tabular}

$\mathrm{P}>0.05$, not significantly difference

Calculation of parasitemia density just started 48 hours after infection. The density of parasitemia of Mus musculus Balb/ $\mathrm{C}$ white mice from the three treatment groups showed the highest density in the control group, followed by the pellet extract group and the lowest in the supernatant extract group. Table 1 known the results obtained were not significantly different ( $P>0.05$ ) from the three treatment groups, both the control group, the pellet extract, and the salivary gland supernatant An. sundaicus with parasitemia density. 


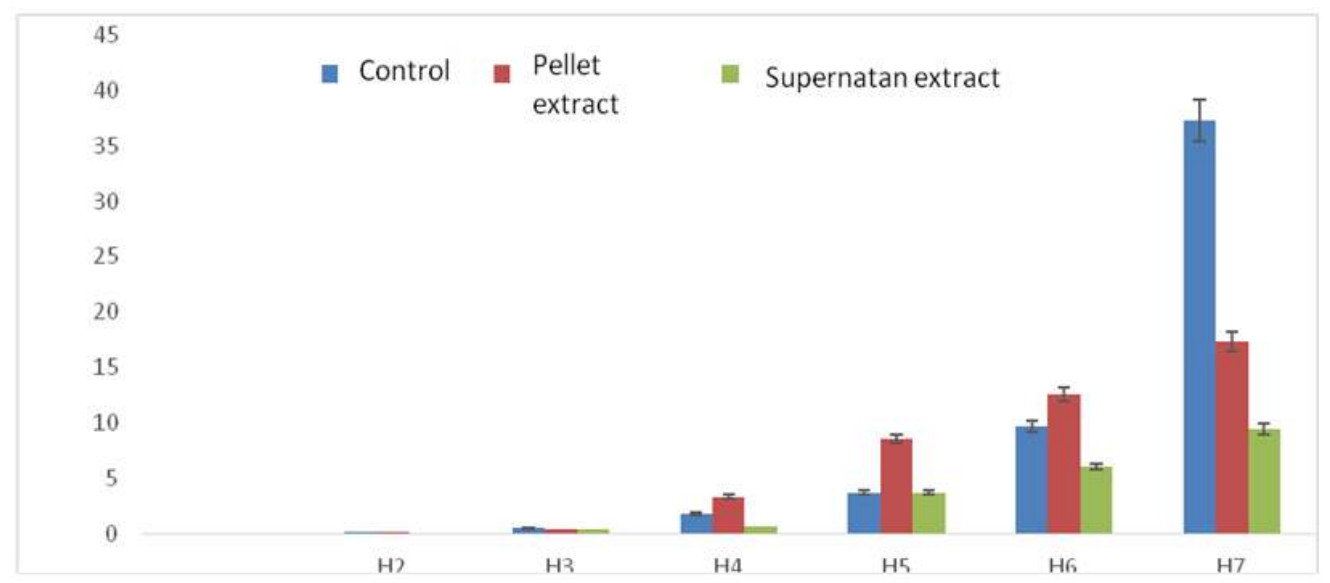

Fig. 2. Development of $P$. berghei parasitemia degree in Mus musculus Balb / C white mice 48 hours post infection until the seventh day of observation

\section{Discussion}

The results showed that the density of parasitemia of Mus musculus Balb / C white mice after infection with $P$. berghei, from the three treatment groups, obtained the highest density in the control group, followed by the pellet extract group and the lowest in the supernatant extract group. It shows that the mice group immunized with supernatant extract had a higher ability to suppress parasitic growth than the mice group immunized with pellet extract.

Based on the results of the ANOVA test and further testing of Tukey-HSD, administration of the salivary glands An. sundaicus in Mus musculus Balb/ $\mathrm{C}$ white mice against parasitemia density showed results that were not significantly different, but in numbers (the average treatment of the three treatment groups) showed the extract of Supernatant salivary gland $A n$. sundaicus was able to reduce $61.94 \%$ to $P$. berghei parasitemia density, while pellet extract was only able to reduce $20.29 \%$ to $P$. berghei parasitemia density compared to the control group. The high percentage reduction in parasitemia density by administering a supernatant extract of salivary glands An. sundaicus indicates that proteins that are immunomodulatory are more soluble.

Repeated exposure to vector mosquito bites with salivary gland antigens causes the host's immune system to generate cellular and/or humoral reactions in the bite site, resulting in rejection of the ectoparasites. The host resistance is related to Th1 immune response with the production of interferon- $\gamma$ (IFN) $-\gamma$, interleukin-2 (IL) -2 and IL-12 [18]. Previous research explains that an increase in IFN- $\gamma$ and IL-12 characterizes the immune response that appears to be more directed to Th- 1 . The increase of cytokinesis parallel to the occurrence of a decrease in some parasitemia in the liver and blood. This shows that protein induction of vector salivary gland extract can protect the host against parasitic infection [13].

Infection of $P$. berghei parasite causes positive feedback because IFN- $\gamma$ produced by Th1 will stimulate dendritic cells or macrophages to produce more IL-12. The high secretion of IL12 will stimulate IFN- $\gamma$ secretion so that the levels of IFN- $\gamma$ in the host's body will be higher. Then this is because the erythrocytes infected with Plasmodium will cause activation of 
macrophage cells to secrete tumor necrosis factor alpha (TNF $\alpha$ ), Nitric Oxide (NO), interleukin 12 (IL-12) to activate NK cells and secrete IFN $\gamma$ [19].

Th1 cells and Th2 cells both contribute to protective immunity to malaria infection, but at different times and the balance between the two determines the manifestation of the disease. Th1 cells are responsible for the control of parasitemia at the beginning of infection, and then Th2 is needed to complete the destruction of parasites. This is demonstrated by the presence of dominant IFN- $\gamma$ production at the beginning of acute $P$. chabaudi infection, then decreases with decreased parasitemia and is subsequently replaced by IL-4 and IL-10 production in advanced stages of infection. Thus the Th1 response followed by Th2 is important to control the development of parasites [20] effectively.

Based on the results of the study on the measured parameters parasitemia density, administration of An salivary gland extracts An. sundaicus is not significantly different from the density of parasitemia. There is no real difference from An salivary gland extract $A n$. sundaicus (pellets and supernatant extract) against parasitemia density, may be caused by many factors. This is in accordance statement that IL-12 plays an important role in the initial response against Plasmodium, and plays a critical role by producing IFN- $\gamma$, which will stimulate the response to Th-1 both in the initial and continuous stages [1].

IL-12 will induce experimental animals with the ability of IL-12 to convert CD4+ to Th1 and will produce monocyte IFN- $\gamma$ to immediately excrete TNF- $\alpha$ which promotes antiplasmodium release such as Nitrix Oxide (NO). Low IL-12 levels in severe malaria indicate that IL-12 is important for immunoprotection against malaria in humans. Based on previous research from which showed barriers that occur in the growth of experimental animal parasites that have previously been sensitized with repetitive sterile mosquito bites involving the cellular adaptive immune system. The high level of Interferon- $\gamma$ as a Th1 product inhibits parasite growth in erythrocytes through the resulting nitrite oxide. Nitric Oxide plays a role in eliminating Plasmodium in hepatocyte cells in the pre-erythrocytic cycle of malaria $[8,13,21]$.

The shift in Th2 levels that benefit the parasite towards Th1 which benefits the host is an influential factor in the development of parasites in erythrocytes. It is also corroborated or relevant to the occurrence of a decrease in the density of parasitemia that can be reduced by administering an Supernatant Extract of the salivary gland. sundaicus was $61.94 \%$ against $P$. berghei parasitemia density, and by pellet extract $20.29 \%$ against $P$. berghei parasitemia density. It shows that the induction of salivary gland protein is An. sundaicus can protect the host against parasitic infection $(P$. berghei).

\section{Conclusion}

Supernatant extracts and salivary gland pellets extract $A n$. sundaicus against white mice Mus musculus Bal / C infected with P.berghei can reduce the density of parasitemia.

\section{References}

[1] Abbas AK, Lichtman AH. Cellular and Molecular Immunology. 5th ed. Elsevier Science, Philadelphia, 2003.

[2] Carter R, Mendis KN, Miller LH, Molineaux L, Saul A. Malaria Transmission Blocking Vaccine: How Can Their Development be Supported. Nat Med. 2000; 6(3): 241-4.

[3] Coutinho-Aabreu IV, Ramalho-Ortigao M. Transmission Blocking Vaccines to Control InsectBorne Diseases-A Review. Mem Inst Oswaldo Cruz; 105 (1): 1-12. 
[4] Lavazec C, Boudin C, Lacroix R, Bonnet S, Diop A, Thiberge S, et al. Carboxypeptidase B of Anopheles gambiaeas Target for a Plasmodium falciparum Transmission-Blocking Vaccine. Infect Immun; 75(4) : 1635-42.

[5] King JG, Vernick KD, Hillyer JF. Members of the salivary gland surface protein family (SGS) are major immunogenic components of mosquito saliva. J Biol Chem. 2011; 286(47): 40824-34.

[6] DeRoeck D, Deen J, Clemens JD. 2003. Policymakers' views on dengue fever/dengue haemorrhagic fever and the need for dengue vaccines in four southeast Asian countries. Vaccine. 2000; 22(1):121-9.

[7] Brennan JDG, Kent M, Dhar R, Fujioka H, Kumar N. Anopheles gambiae Salivary Gland Proteins as Putative Targets for Blocking Transmission of Malaria parasites. Proc Natl Acad Sci U S A. 2000; 97(25): 13859-13864.

[8] Titus RG, Bishop JV, Mejia JS. The immunomodulatory factors of arthropod saliva and the potential for these factors to serve as vaccine targets to prevent pathogen transmission. Parasite Immunol. 2006;28(4):131-41.

[9] Chattopadhyay R, Kumar S. Malaria Vaccine: Latest update and challenges ahead. Indian J Exp Biol. 2009;47(7):527-36

[10] Ramirez JL, Garver LS, Dimopoulos G. Challenges and Approaches for Mosquito Targeted Malaria Control. Curr Mol Med. 2009;9(2):116-30

[11] Wasinpiyamongkol L, Patramool S, Luplertlop N, Surasombatpattana P, Doucoure S, Seveno $\mathrm{M}$, et al. Blood feeding and imunogenic aedes aegypti saliva proteins. Proteomic. 2010; 10(10): 1906-1916.

[12] Wongkamchai S, Khongtak P, Leemingsawa S, Komalamisra N, Junsong N, Kulthanan K, et al. Comparative identification of protein profiles and major allergens of saliva, salivary gland and whole body extracts of mosquito species in Thailand. Asian Pac j Allergy Immunol. 2010; 28: $162-9$

[13] Donovan MJ, Messmore AS, Scrafford DA, Sacks DL, Kamhawi S, McDowell MA. Uninfected mosquito bites confer protection against infection with Malaria parasite. Infect Immun. 2007; 75(5) : 2523-30.

[14] Kamhawi S, Belkaid Y, Modi G, Rowton E, Sacks D. Protection against cutaneus Leishmaniasis resulting from bite of uninfected sand flies. Science. 2000. 290(5495): 1351-4.

[15] Gardiner DL, McCarthy JS, Trenholme KR. Malaria in the post-genomics era: light at the end of the tunnel or just another train? Postgrad Med J. 2005; 81:505-9.

[16] World Health Organization. Division of Malaria and Other Parasitic Diseases. Manual on Practical Entomological Field Techniques For Malaria Control. WHO, Geneva, 1975.

[17] Omar SH, el-Matarawy OM, el-Naggar SM, Abdel Salam NM, el-Rehemi HH. The course of Plasmodium berghei infection in mice subjected to variable dosages of the parasite and the peak parasitaemia obtained accordingly. J Egypt Soc Parasitol. 1986 ;16(2):757-61

[18] Andrade BB, Teixeira CR, Barral A, Barral-Netto M. 2005. Haematophagous arthropod saliva and host defense system: a tale of tear and blood. An Acad Bras Cienc. 2005; 77(4): 665-93.

[19] Schofield L, Grau GE. Immunological processes in malaria pathogenesis. Nat Rev Immunol. 2005;5(9):722-35.

[20] Wipasa J, Elliot S, Xu H, Good MF. Immunity to Asexual Blood Stage malaria and Vaccine Approaches. Immunol Cell Biol. 2002;80(5):401-14.

[21] Angulo I, Fresno M. Cytokines in the pathogenesis of and protection against malaria. Am Soc for Microbiology. 2002; 9(6):1145-52. 